\title{
In vitro Activities of Antimicrobial Agents against Uropathogenic Isolates at Brong Ahafo Regional Hospital, Ghana
}

\author{
Solomon Wireko ${ }^{1,2}$, Amina Abubakari ${ }^{2} *$ and Bempah Opoku ${ }^{2}$ \\ ${ }^{1}$ Ejisu Government Hospital, Ejisu-Juaben Municipal Assembly-Ghana \\ ${ }^{2}$ Department of Laboratory Technology, Faculty of Health Sciences, Kumasi Technical \\ University, Kumasi- Ghana \\ *Corresponding author
}

\section{A B S T R A C T}

\section{Keywords}

Enterobacteriaceae,

In vitro,

Antimicrobial,

Uropathogenic.

Article Info

Accepted:

04 April 2017

Available Online:

10 May 2017
Bacterial resistance against antimcrobial agents is a growing international problem in the treatment of nosocomial infections, especially in developing countries. This study therefore sought to determine the antibiotic susceptibility pattern of the isolates in UTIs and to identify the probable antibiotic alternatives in uropathogenic infection to guide antimicrobial therapy at the Brong-Ahafo Regional Hospital-Sunyani. Between January and December 2014, 200 urinary isolates were received from in and out patients at the Microbiology unit of the laboratory. Isolates were tested for antimicrobial susceptibility by the Kirby Bauer disc diffusion method, on Mueller-Hinton Agar (Oxoid GmbH, Wesel, Germany) and the results were presented as resistant or susceptible, according to the recommendations of Clinical and Laboratory Standards Institute (CLSI). One hundred and seventy-two $(172,96.6 \%)$ isolates were resistant to tetracycline and $144(90.5 \%)$ isolates were resistant to Ampicillin/sulbactam. One hundred and fifty $(150,93.2 \%)$ isolates were readily susceptible to Amikacin. Among the third generation cephalosporins, ceftizoxime achieved $50 \%$ sensitivity and $20.6 \%$ and $16.7 \%$ for cefotaxime and ceftazidime respectively against all isolates. The isolates also showed strong resistance to the fluoroquinolones, nalidixic acid $(80.8 \%)$; ciprofloxacin $(74.1 \%)$; ofloxacin $(65.4 \%)$ and levofloxacin (64.6\%). Chloramphenicol and gentamicin achieved $23.4 \%$ and $24.1 \%$ sensitivity respectively. Lower resistance was observed in amikacin and ceftizoxime. The extent of resistance among bacteria isolates in UTIs in non-hospitalized and hospitalized patients is worryingly high in the Brong-Ahafo Regional Hospital. Antimicrobials such as tetracycline, ampicillin/sulbactam, chloramphinicol and gentamicin should no longer be recommended for initial empirical therapies for UTIs especially when $E$. coli is concerned. Amikacin, ceftizoxime, ofloxacin and levofloxacin may be considered as alternatives.

\section{Introduction}

Antibiotic are among the commonly administered drugs in many hospitals and over dependence on antibiotics is regarded among the many reasons for the rising trend of resistance observed among different pathogens (Gonçalves et al., 2016). All over the world grave concern has been expressed about the increase in the numbers of organisms that are multi-drug resistant (HimaLerible et al., 2003; Howladar and Gandhi, 2016) and the difficulty encountered in their treatment. The high increase in numbers of 
infections due to antibiotic-resistant bacteria complicates their treatment and may even threaten patients' lives (Fashad et al., 2010; Steinke et al., 2001). The World Health Organization (WHO) and the European Commission (EC) have acknowledged the necessity of analyzing the emergence and determining factors leading to resistance and the importance of strategies towards its control (Eryilmaz et al., 2010; Kollef and Fraser, 2001; WHO, 2002). In Ghana, increasing rate of resistance against different antibiotic classes have been observed among many bacterial pathogens (Newman et al., 2011; Opintan and Newman, 2007; MillsRobertson, 2003).

Implicated factors include ease of access to antimicrobials leading to increase in consumption of antibiotics by humans and animals ( $\mathrm{Li}$ et al., 2007) giving rise to increased selection pressure on the gene pool for antimicrobial resistance (Huttner et al., 2013)

Want of resources which seem to defeat the discharge of WHO intervention programs like putting in place a national task force, formulating indices to supervise and appraise the consequence of antimicrobial resistance, and planning microbiological reference facilities that would organize efficient close observation of resistance to antimicrobials among usual pathogens (WHO, 2001). Furthermore, in several third world countries interventions using alternative agents may be limited and out of reach of most patients.

An evaluation of antibiotic resistance in Ghana found staggeringly high prevalence of resistance to these common antibiotics: chloramphenicol, 75\%; tetracycline, $82 \%$ cotrimoxazole 72\%; among Gram-negative bacteria isolated from in-patients: $E$. coli: ampicillin, 75\%; cefotaxime, $20 \%$ and nalidixic acid, 49\%. Similar resistance prevalence was recorded in other gastrointestinal pathogens including Salmonella spp. which may also cause bacteraemia (Newman et al., 2006). Data obtained from the Komfo Anokye Teaching Hospital laboratory record books showed that among Out- patients there is substantial resistance to antimicrobials involving several of the Enterobacteriaceae to even the third generation cephalosporins (Feglo, 2007).

Urinary tract infection (UTIs) refers to microbial colonization of the urine and tissue invasion of the urinary tract mostly by bacteria, though viruses and yeast may be involved (Schaeffer and Schaeffer, 2007).

UTIs, as a common hospital and communityacquired bacterial infection, affect all age groups. The prevalence rate among infants is $6.5 \%$ and $3.3 \%$ among girls and boys respectively (Bressan et al., 2009). It is the second most usual cause of infectious disease related hospitalization among adults aged 65years and beyond (Curns et al., 2005).

Several studies have revealed gram-negative bacilli: Escherichia coli and Salmonella typhi, Pseudomonas aeruginosa, Klebsiella pneumoniae, as the predominant bacterial isolates from UTIs (Ophori et al., 2010; Alebiosu et al., 2003). Escherichia coli is the most prevalent facultative anaerobic species in the human gastrointestinal tract $\left(10^{5} \mathrm{CFU} / \mathrm{g}\right.$ faeces), and it is known to be the cause of about $75-90 \%$ of all cases of UTIs among inpatients and outpatients (Dromigny et al., 2005, Marrs et al., 2005; Ejrnaes et al., 2006; Johnson and Russo, 2005).

Urinary tract infections (UTI's) caused by antimicrobial resistant bacteria, especially ESBL-producing Enterobactriaceae, can be life threatening as therapeutic options available to treat infected patients are limited. This study therefore sought to determine the 
antibiotic susceptibility pattern of the uropathogenic isolates in UTIs and to identify the probable antibiotic alternatives to guide antimicrobial therapy at the Brong-Ahafo Regional Hospital-Sunyani.

\section{Materials and Methods}

\section{Study area}

The study was conducted at the Brong Ahafo Regional Hospital, which is a 300 bed and secondary referral hospital in the Brong Ahafo Region, with population of about two million inhabitants. The Region has 19 districts with Sunyani as the Regional capital.

\section{Study design}

This was a cross-sectional study involving 200 samples. Urine samples for the study were obtained from patients referred to the Microbiology Laboratory of the hospital for diagnosis. Patients who reported to the hospital and were suspected of UTI and who gave their informed consent were included in the study.

\section{Ethical clearance}

Ethical clearance was obtained from the Institutional Ethics Committee of the BrongAhafo Regional Hospital, Sunyani.

\section{Bacterial isolation}

Mid-stream urine samples were received from patients at the Microbiology laboratory and plated on cysteine lactose electrolyte deficient (CLED) agar using a 1/400 $\mu \mathrm{m}$ calibrated loop and pure colonies of $\geq 25$ growing on CLED agar after overnight incubation at $37^{\circ} \mathrm{C}$ were considered significant for diagnosis of urinary tract infection. This is equivalent to growth of $10^{5}$ colony forming units/ml of one organism type and was considered significant bacteriuria. Where there were mixed growth of different organisms it was considered contaminations and were rejected. The growths were identified by their colonial growth morphology on CLED medium. Lactose fermenting colonies suspected to be E. coli or Klebsiella pneumoniae were tested further using Gram stain (Gram negative rods), the motility test and then indole and methyl red, citrate and Voges-Proskauer tests.

Non lactose fermenters that could be Proteus, Providencia, Morganella or Salmonella species were also further tested using Gram stain and biochemical tests including indole production, citrate utilization, and urease production before definitive identification.

\section{Antibiotic susceptibility testing}

Antibiotic sensitivity of the isolates was determined by the modified Kirby-Bauer method according the CLSI recommended guidelines (Vol. 32, 20th edition): Approved Standard M02-A11 (CLSI, 2012).

Each set of tests was controlled using susceptible E. coli control strain (ATCC 25922). Antimicrobials and their concentrations tested included: ampicillin/sulbactam 20ug, amikacin 30ug, cefotaxime $30 \mathrm{ug}$, ceftazidime 30ug, chloramphenicol 30ug, ciprofloxacin 5ug, gentamicin 10ug, ofloxacin 5ug, nalidixic acid 10ug, tetracycline 30ug, Levofloxacin 5ug, and ceftizoxime $30 \mathrm{ug}$.

\section{Data processing and analysis}

Continuous data were expressed as mean \pm SD and categorical data expressed as proportion. Resistance proportions were compared using chi-square tests. In all cases a p-value $<0.05$ was considered significant. The data were analyzed using Stata/IC 10.0 for windows (StataCorp LP, USA, http://www.stata.com). 


\section{Results and Discussion}

\section{Characteristics of study participants}

Table 1 gives the general sociodemographic characteristics of the study population. Patient ages ranged between 5 months and 92 years old. There were 132 adults above 18 years and 57 children from 1 month to 18years. One hundred and thirteen $(56.5 \%)$ of the study participants were males and 87 (43.5) were females.

Prevalence and distribution of uropathogens

During the study period, January to December, 2014, 1,302 urine samples were received and a total of 200 non-duplicate uropathogens were isolated from both inpatients 131(65.5\%) and out patients $69(34.5 \%)$, giving a prevalence of $15.4 \%$ (200/1302) and distributed as shown in table 2 .

\section{Antibiotic susceptibility pattern of the isolates}

Tetracycline and ampicillin/sulbactam had the lowest sensitivity, $172(96.6 \%)$ isolates were resistant to tetracycline and 144(90.5\%) isolates were resistant to ampicillin/ sulbactam. One hundred and fifty $(93.2 \%)$ isolates were readily susceptible to amikacin. Among the third generation cephalosporins, ceftizoxime achieved $50 \%$ sensitivity and $20.6 \%$ and $16.7 \%$ for cefotaxime and ceftazidime respectively as shown in (Table $3)$.

The isolates also showed high resistance proportions to the fluoroquinolones, nalidixic acid (80.8\%); ciprofloxacin $(74.1 \%)$; ofloxacin $(65.4 \%)$ and levofloxacin $(64.6 \%)$. Chloramphenicol and gentamicin achieved 23.4 and $24.1 \%$ sensitivity respectively.

Table.1 General characteristics of study participant

\begin{tabular}{lcc}
\hline Variables & No & $(\%)$ \\
\hline Age groups (years) & 132 & 66.0 \\
Adults above 18years & 57 & 28.0 \\
Children (1 month-18yrs) & 11 & 6.0 \\
Unknown & & \\
& & \\
Gender & 113 & 56.5 \\
Male & 87 & 43.5 \\
Female & & \\
WARD & 131 & 65.5 \\
IN-PATINET & 69 & 34.5 \\
OPD & & \\
\hline
\end{tabular}


Table.2 Distribution of uropathogenic bacterial isolates stratified by in-patient and out-patients

\begin{tabular}{llcc}
\hline Organism in UTI & TOTAL & IN-PATIENT & OUT-PATIENT \\
& N $(\%)$ & $\mathbf{N}(\%)$ & $\mathbf{N}(\%)$ \\
\hline Citrobacter spp. & $34(17.0 \%)$ & $22(64.7 \%)$ & $12(35.3 \%)$ \\
Coagulase negative staph. & $12(6.0 \%)$ & $7(58.3 \%)$ & $5(41.7 \%)$ \\
E. coli & $51(26.0 \%)$ & $31(60.8 \%)$ & $20(39.2 \%)$ \\
Enterobacter & $40(20.0 \%)$ & $21(52.5 \%)$ & $19(47.5 \%)$ \\
Enterococcus faecalis & $2(1.0 \%)$ & $2(100.0 \%)$ & $0(0.0 \%)$ \\
Klebsiella spp & $5(2.5 \%)$ & $3(60.0 \%)$ & $2(40.0 \%)$ \\
Moganella morganii & $2(1.0 \%)$ & $1(50.0 \%)$ & $1(50.0 \%)$ \\
Proteus mirabilis & $3(1.5 \%)$ & $2(66.7 \%)$ & $1(33.3 \%)$ \\
Proteus vulgaris & $1(0.5 \%)$ & $1(100.0 \%)$ & $0(0.0 \%)$ \\
Providencia spp & $7(3.5 \%)$ & $6(85.7 \%)$ & $1(14.3 \%)$ \\
Pseudomonas aeruginosa & $8(4.0 \%)$ & $7(87.5 \%)$ & $1(12.5 \%)$ \\
Salmonella typhi & $1(0.5 \%)$ & $1(100.0 \%)$ & $0(0.0 \%)$ \\
Serratia marcescens & $6(1.5 \%)$ & $5(83.3 \%)$ & $1(16.7 \%)$ \\
Staphylococcus aureus & $12(6.0 \%)$ & $9(75.0 \%)$ & $3(35.0 \%)$ \\
Streptococcus spp. & $7(3.5 \%)$ & $5(71.4 \%)$ & $2(25.6 \%)$ \\
others & $9(4.5 \%)$ & $6(66.7 \%)$ & $3(33.3 \%)$ \\
Total & $200(100.0 \%)$ & $131(65.5 \%)$ & $69(34.5 \%)$ \\
\hline
\end{tabular}

Table.3 Frequency of antibiotic sensitivity and resistance pattern

\begin{tabular}{lcc}
\hline Antibiotics & Sensitivity of all isolate & Resistance of all isolate \\
\hline Ampicillin/Sulbactam (As) & $15(9.4 \%)$ & $144(90.5 \%)$ \\
Nalidixic Acid (Nx) & $27(19.3 \% 0$ & $113(80.8 \%)$ \\
Cefotaxime (Ctx) & $13(20.0 \%)$ & $50(79.4 \%)$ \\
Ceftazidime (Caz) & $9(16.7 \%)$ & $45(83.3 \%)$ \\
Chloramphenicol (Ch) & $37(23.4 \%)$ & $121(76.6 \%)$ \\
Ciprofloxacin (Cp) & $45(25.9 \%)$ & $129(74.1 \%)$ \\
Ceftizoxime (Cl) & $63(50.0 \%)$ & $63(50.0 \%)$ \\
Tetracycline (Te) & $6(3.4 \%)$ & $172(96.6 \%)$ \\
Ofloxacin (Of) & $56(34.6 \%)$ & $106(65.4 \%)$ \\
Gentamicin (Gm) & $13(24.1 \%)$ & $41(75.9 \%)$ \\
Amikacin (Ak) & $150(93.2 \%)$ & $11(6.8 \%)$ \\
Levofloxacin (Le) & $51(35.4)$ & $93(64.6 \%)$ \\
\hline
\end{tabular}

This study found prevalence of UTI to be $15.4 \%$, with the uropathogens distributed across all age groups. The most implicated organisms were gram negatives including $E$. coli, Enterobacter, Pseudomonas, Proteus, Klebsiella species, Citrobacter and few gram positives including $S$. saprophyticus and $S$. aureus. E coli was the highest isolate. This finding is in agreement with previous studies (Moyo et al.,2010; Dromigny et al., 2005; Ophori et al.,2010; Alebiosu et al., 2003), in which similar organism were isolated from 
UTI with $E$. coli as the highest isolate. $E$. coli has also been noted to be more frequently isolated from females than from males (Motayo et al., 2012), a pattern that was also observed in this study with 33 females and 18 males $(35 \%)$ infected with E. coli. This observation could be due to anatomical differences in the genitalia that predisposes females to UTI (Puri and Malhotra, 2009).The current study observed a high prevalence of antimicrobial resistance against all the regular antibiotics in use at the hospital. Multidrug resistance (MDR) was observed among all the uropathogens. Extensively drug resistance (XDR) was observed among E. coli, Klebsiella spp. and Staphylococcus aureus but none of the uropathogens exhibited Pan drug resistance (PDR). The highest level of resistance was against tetracycline (96.6\%), ampicillin/ sulbactam (93.2\%), chloramphenicol (76.6\%), and gentamicin $(75.9 \%)$. These levels of resistance are comparable to levels obtained in a previous study in Ghana that also had similar high levels of resistance (Newman et al., 2011), however the levels were higher than that obtained from Senegal (Dromigny et al., 2005), Tanzania (Eryimaz et al., 2010), Canada and USA (Zhanel et al., 2006). These differences could be due to various reasons including the fact that the rate of resistance had been rising over the years. The differences in the resistance rates to same antibiotics in the three studies support the submission by a previous study that Empirical therapy should be based on local antimicrobial resistance monitoring in order to prevent increase in resistance to drugs used in the treatment of UTIs (Kutlu and Kutlu, 2007). Knowledge on local antimicrobial resistance trends among urinary isolates is important in guiding clinicians to prescribe appropriate antibiotics and also for evidence based recommendations in empirical antibiotic treatment of UTI. Recent studies have shown that previously effective classes of antibiotics such as the cephalosporins and fluoroquinolones have experienced serious setbacks in emperical therapy (Newman et al., 2011; Rice, 2012). The present study demonstrated same high levels of resistance to the fluoroquinolones: nalidixic acid (80.8\%); ciprofloxacin (74.1\%); ofloxacin $(65.4 \%)$ levofloxacin $(64.6 \%)$ and third generation cephalosporins: ceftazidime, cefotaxime and ceftizoxime. The least resistance was found in Amikacin (6.8\%). Amikacin and nitrofurantoin were found to be the most effective treatments for UTIs in China and India, according to Shao et al., (2004) and Mandira Mukherjee et al., (2013) respectively. This means amikacin could still be used as effective treatment for UTIs in Ghana.

The high resistant proportions seen in this current study is attributable to many factors. Firstly, many hospitals in Ghana do not have the competence and the facility to carry out bacterial culture, isolation and antimicrobial sensitivity testing, so physicians prescribe drugs to patients based on symptomatic evidence without laboratory support. The second factor is the indiscriminate use of the drugs, because they are relatively cheap and easily accessible by the patient. The drugs can be purchased off the counter due to lack of enforcement of regulations, and also because the antibiotics are taken orally, so they are easy to administer and misuse (Newman et al., 2006). Another link to antibiotic resistance is the increased use of antibiotics in agriculture in animal husbandry coupled with the production and sale of substandard drugs (Tajick, 2006; Shakoor et al., 1977).

In conclusion this study, has demonstrated that the prevalence of bacterial isolates from UTI is high and that most of the pathogens isolates are highly resistant to the usual antimicrobials used in their treatment in the Regional Hospital. The highest level of 
resistance was observed against tetracycline, followed by ampicillin/sulbactam and on the basis of the findings from this study, antimicrobials such as tetracycline, ampicillin/sulbactam, chloramphenicol and gentamicin should no longer be recommended for initial empirical therapies for UTIs especially when $E$. coli is concerned. The drugs with lowest resistance were amikacin, ceftizoxime, ofloxacin and levofloxacin and therefore may be considered as alternatives but before such a decision, the antimicrobial susceptibilities of the pathogens causing the UTIs should be investigated and necessary precautions taken against resistance development.

Based on the findings of this study it is therefore recommended that; antimicrobial agent usage policies, especially empirical therapies, should be based on antimicrobial resistance surveillance studies, regular data should be collected on emerging resistance to new antimicrobial drugs such as tigecycline and colistin, for quick and easy tracking and also clinical laboratories should be well resourced and well trained to carry out bacterial culture, isolation and antimicrobial sensitivity.

\section{References}

Alebiosu, C.O., Osinupebi, O.A., Olajubu, F.A. 2003. Significant asymptomatic bacteriuria among Nigerian type two diabetics. J. Natl. Med. Assoc., 95: 344351.

Bressan, S., Andreola, B., Zuchetta, P. 2009. Procalcitonin as a predictor of renal scarring in infants and young children. Pediatrick Nephrol., 24(6): 1199-204.

CLSI. 2012. Performance Standards for Antimicrobial Disk Susceptibility Tests; Vol. 32(20th edition): Approved Standard M02-A11

Curns, A.T., Holman R.C., Sejvar J.J.,
Owings MF, Schongberger L.B. 2005. Infectious disease hospitalizations among older adults in the United States from 1990 through 2002. Arch. Int. Med., 165(21): 2514-20.

Dromigny, J.A., Nabeth, P., Juergens-Behr, A., Perrier- Gros-Claude, J.D. 2005. Risk factors for antibiotic resistant Escherichia coli isolated from community-acquired urinary tract infections in Dakar, Senegal. $J$. Antimicrob. Chemother., 56: 236-239.

Ejrnaes, K., Sandvang, D., Lundgren, B., Ferry, S., Holm, S., Monsen, T., Lundholm, R. 2006. Pulsed field gel electrophoresis typing of Escherichia coli strains from samples collected before and after pivmecillinam or placebo treatment of uncomplicated community acquired urinary tract infection in women. J. Clin. Microbiol., 44: 1776-1781.

Eryılmaz, M., Merve, E. B., Muharrem, M. Y., Ahmet, A. 2010. Antimicrobial Resistance of Urinary Escherichia coli. Isolates. Trop. J. Pharm. Res., 9(2): 205.

Farshad, S., Reza, R., Mojtaba, A., Maneli, A.S., Marziyeh, H. 2010. Emergence of Multi Drug Resistant Strains of Eschetichia coli Isolated from Urinary Tract Infection. The Open Conference Proceedings J., 1: 192-196.

Feglo, P. 2007. Antimicrobial sensitivity patterns of urine isolates at the KomfoAnokye Teaching Hospital, (KATH) Kumasi, 2000-2005. Ghana J. Allied Health Sci., 1(2): 43-49.

Gonçalves, L.F., de Oliveira Martins-Júnior, P., de Melo, A.B.F., da Silva, R.C.R.M., de Paulo Martins, V., Pitondo-Silva, A., and de Campos, T.A. 2016. Multidrug resistance dissemination by extendedspectrum $\quad \beta$-lactamase-producing Escherichia coli causing communityacquired urinary tract infection in the 
Central-Western Region, Brazil. J. Global Antimicrobial Resistance, 6: 1-4. Hima-Lerible, H., Ménard, D., Talarmin, A. 2003. Antimicrobial resistance among uropathogens that cause communityacquired urinary tract infections in Bangui, Central African Republic. $J$ Antimicrob. Chemother., 51: 192-194.

Howladar, A.A., and Gandhi, P. 2016. A Study To Determine Prevalence of Quinolone Resistance Genes Among Extended-Spectrum B-LactamaseProducing Escherichia coli. Global J. Res. Analysis, 5.

Huttner, A., Harbarth S., Carlet, J., Cosgrove, S., Goossens, H., Holmes, A., Jarlier, V., Voss, A. and Pittet, D. 2013. Antimicrobial resistance: a global view from the 72 .

Johnson, J.R., Russo, T.A. 2005. Molecular epidemiology of extra intestinal pathogenic (uropathogenic) Escherichia coli. J. Med. Microbiol., 295: 383-404.

Kollef, M.H. and Fraser, V.J. 2001. Antibiotic Resistance in the Intensive Care Unit. Annals of Internal Med., 134(4): 298314.

Kutlu, S., Kutlu, M. 2007. Antibiotic susceptibility of Escherichia coli strains isolated in urinary tract infections in Didim, Turkey. Turk. J. Infect., 21(2): 81-83.

Li, Q., Sherwood, J.S., Logue, C.M. 2007. Characterization of antimicrobial resistant Escherichia coli isolated from processed bison carcasses. J. Appl. Microbiol., 103: 2361-2369.

Mandira Mukherjee, ShreyaBasu, Sandip Kumar Mukherjee, Monalisa Majumder. 2013. Drug resistance in uropathogenic E. coli. J. Clin. Diag. Res., 7(3): 449-453.

Marrs, C.F., Zhang, L., Foxman, B., Wicher, K., Kosscinski, D. 2005. Escherichia coli mediated urinary tract infection: are there distinct uropathogene $E$. coli
(UPEC) pathotypes culture media. FEMS Microbiol. Lett., 252: 183-189.

Mills-Robertson, F., Crupper, S. S., Addy, M. E., Mensah, P. 2003. Antibiotic resistance and genotyping of clinical group B Salmonella isolated in Accra, Ghana. J. Appl. Microbiol., 94(2): 289294.

Motayo, O.B., Ogiogwa, I.J., Okerentugba, P.O., Innocent-Adiele, H.C., Nwanze, J.C., Onoh, C.C. and Okonko, I.O. 2012. "Antimicrobial Resistance Profile of Extra-Intestinal Escherichia coli Infections in a South Western Nigerian City. J. Microb. Res., 2(5): 141-144.

Moyo, S.J., Said, A., Mabula, K., Eligius, F.L. and Samuel, Y.M. 2010. Antimicrobial resistance among producers and non-producers of extended spectrum beta lactamases in urinary isolates. BMC Res. Notes, 3: 348.

Newman, M.J., Frimpong, E. Donkor, E.S., Opintan, J.A, Asamoah-Adu, A. 2011. Resistance to antimicrobial drugs in Ghana. Infect and Drug Res., 4: 215220.

Newman, M.J., Frimpong, E., Asamoah-Adu, A., Sampene-Donkor, E. 2006. Resistance to antimicrobial drugs in Ghana. The Ghanaian-Dutch Collaboration for Health research and Development. Project number 2001/GD/07. Technical report series, No. 5: 8-26.

Ophori, E.A., Imade, P., Johnny, E.J. 2010. Asymptomatic bacteriuria in patients with type 2 diabetes mellitus. J. Bact. Res., 2(22): 14-17.

Opintan, J.A., Newman, M.J. 2007. Distribution of serogroups and serotypes of multiple drug resistant Shigella isolates. Ghana Med. J., 41(1): 50-54.

Puri, R. and Malhotra, J. 2009. Recurrent Urinary Tract Infection (UTI) in 
Women. South Asian Federation of Obstetrics and Gynecol., 1(1): 10-13.

Rice, B.L. 2012. Mechanisms of Resistance and Clinical Relevance of Resistance to $\beta$-Lactams, Glycopeptides, and Fluoroquinolones. Mayo Clin. Proc., 87(2): 198-208.

Schaeffer, A.J., Schaeffer, E.M. 2007. Infection of the Urinary Tract, In: A. J. Wein, et al., Eds, Campbell- Walsh Urology, 9th Edition, Saunders, Philadelphia, pp. 223-303.

Shakoor, O., Taylor, R. B., \& Behrens, R. H. 1977. Assessment of the incidence of substandard drugs in developing countries. Trop. Med. Int. Health, 2(9): 839-845

Steinke, D.T., Seaton, R.A., Phillips, G., MacDonald, T.M. and Davey, P.G. 2001. Prior trimethoprim use and trimethoprim-resistant urinary tract infection: a nested case-control study with multivariate analysis for other risk factors". J. Antimicrob. Chemother., 47: 781-787.

Tajick, M.A. 2006. Detection of antibiotic residue in chicken meat using TLC. Intl. J. Poultry Sci., 5(7): 611-612.

WHO. 2001. Global Strategy for Containment of Antimicrobial Resistance (WHO/CDC/CSR/DRS/2001.2a.

Geneva, Switzerland: World Health Organization.

WHO. 2002. Surveillance standards for antimicrobial resistance. Communicable Disease Surveillance Response, 2-3.

Zhanel, G.G., Hisanaga, T.L., Laing, N.M., De Corby, M.R., Nichol, K.A., Weshnoweski, B., Johnson, J., Noreddin, A., Low, D.E., Karlowsky, J.A., Hoban, D.J. 2006. Antibiotic resistance in Escherichia coli outpatient urinary isolates: final results from the North American Urinary Tract Infection Collaborative Alliance (NAUTICA. Int. J. Antimicrob. Agron., 27(6): 468-475.

\section{How to cite this article:}

Solomon Wireko, Amina Abubakari and Bempah Opoku. 2017. In vitro Activities of Antimicrobial Agents against Uropathogenic Isolates at Brong Ahafo Regional Hospital, Ghana. Int.J.Curr.Microbiol.App.Sci. 6(5): 193-201. doi: http://dx.doi.org/10.20546/ijcmas.2017.605.023 